\title{
Il paesaggio invisibile. \\ La costruzione monastica del territorio negli interstizi del Veneto contemporaneo
}

\author{
di Matteo Melchiorre
}

\author{
$[\ldots]$ \\ per mantenere l'eco di un'armonia \\ $[\ldots]$ \\ A. Zanzotto, Mentre tanfo e grandine e cumuli di guerra \\ in Isola dei Morti - Sublimerie
}

San Benedetto delle Selve, provincia di Padova, comune di Teolo, può essere facilmente sorvolato tramite Google Maps. L'occhio del sorvolatore non faticherà certo a riconoscervi il limpido sigillo paesaggistico del Veneto contemporaneo. Ritagli di prati e campi di pianura (scaleni, di tonalità diverse), qualche isolato residenziale (discosto dal nucleo più vecchio dell'abitato) e infine il segno più netto: la zona industriale con il suo rigore geometrico, raccolta in se stessa come un villaggio, in una scacchiera di grigi ${ }^{1}$.

All'ingrosso, si possono contare 53 capannoni. Bitubo srl. Criocabin spa. Sanifarma. Statmec. Execo srl. Fip chemicals srl. Lavanderia Campesato. Mill Service. Metal Finish. Tecno steel. San Benedetto delle Selve, per intendersi, è il Veneto descritto nei versi sofferenti di Andrea Zanzotto (1999; 2009; 2013), narrato nella prosa sbigottita di Vitaliano Trevisan $(2002 ; 2016)$, interpretato negli studi geografici di Francesco Vallerani e Mauro Varotto (2005; Vallerani, 2013, in particolare pp. 133-182), analizzato nei lavori di non pochi urbanisti (Indovina et al., 1990; Savino, 1999; Munarin e Tosi, 2001; Indovina, 2009); il Veneto, insomma, fisicamente costruito, o per meglio dire affastellato, nel corso di non pochi decenni di euforia imprenditoriale.

Questo Veneto, come noto, è fulmineamente cresciuto imponendosi sopra un assetto paesaggistico fondamentalmente agrario. Ma anche quest'ultimo paesaggio scomparso, che stupì per la sua bellezza viaggiatori come Hippolyte Taine (1993, p. 407) o Johann Wolfgang Goethe (1983, pp. 20-66), fu il frutto di una costruzione. Torniamo, a questo proposito, a San Benedetto delle Selve e alla sua area industriale.

${ }^{1}$ Il progetto di ricerca da cui è nato questo contributo, dal titolo Tra natura e storia: alla ricerca di una diversa strategia per la sostenibilità, è stato sostenuto e finanziato dal Dipartimento di Progettazione e Pianificazione in Ambienti complessi dell'Università IUAV di Venezia, sotto la guida di Laura Fregolent. Nelle pagine che seguono, che vogliono essere una riflessione generale sui punti chiave delle risultanze scientifiche desunte dal lavoro di ricerca, si ometteranno i rinvii puntuali alle molte fonti archivistiche e bibliografiche consultate, delle quali si darà ampio conto in un saggio di prossima pubblicazione.

Archivio di Studi Urbani e Regionali, XLVIII, 119, 2017 - 2017 ISSN 0004-0177 ISSNe 1971-8519 DOI: 10.3280/ASUR2017-119010 
Se avessimo sorvolato quest'area sette secoli fa, avremmo visto una distesa di boschi (le Selve del toponimo). Ma in mezzo a questi boschi avremmo scoperto una radura: alberi abbattuti, ceppi sradicati, contadini con zappe e scuri; forse anche degli aratri, ma sicuramente legname da opera, pietre, sabbia, calcina. Il 27 marzo 1300, infatti, si presentarono dinanzi all'abate di Praglia, la ricca abbazia benedettina dei Colli Euganei, sette uomini. Chiesero che venisse loro concesso un cospicuo terreno del monastero. Si trattava di un fondo «sterile et incultum», posto in una contrada detta Silva Maiore, subito a nord dei Colli Euganei. I sette supplicanti si impegnavano non solo a disboscarlo, a dissodarlo e in progresso di tempo a coltivarlo, ma anche ad abitarlo di persona, costruendovi e fondandovi le proprie abitazioni. Il nuovo abitato ritagliato tra i boschi venne chiamato $\mathrm{S}$. Benedetto delle Selve ${ }^{2}$.

Per costruire una nuova pagina di paesaggio, va da sé, bisogna cancellare, almeno parzialmente, la pagina precedente. Il criterio estetico, in tutto questo, è questione ampiamente secondaria. Nel 1300 a San Benedetto delle Selve i monaci di Praglia e i loro fittavoli cancellarono i boschi, costruirono un paesaggio agrario e fondarono un villaggio assecondando gli interessi economici del tempo. Trascorsi 700 anni, in risposta a nuove e diversissime esigenze economiche, il paesaggio agrario la cui origine era dovuta ai monaci ha ceduto il passo all'odierno paesaggio industriale.

Il caso di San Benedetto delle Selve testimonia in primo luogo come il territorio sia un palinsesto di rasature e riscritture. In secondo luogo porta in primo piano dei "costruttori" di paesaggio, gli enti monastici, che per il dinamismo, per l'estensività e per l'organicità dei loro interventi ebbero una parte fondamentale nella formazione di quel paesaggio storico, oggi pressoché invisibile, sul quale è venuta imponendosi l'ubiqua uniformità urbanistica del Veneto contemporaneo.

Sebbene nel campo degli studi si contrappongano ancora posizioni che sostengono con toni entusiastici il ruolo avanguardistico svolto dai monaci nella formazione del paesaggio e altre che minimizzano e ridimensionano l'effettiva incidenza dell'azione monastica sul territorio ${ }^{3}$, ripercorrere in senso inverso le stratigrafie paesaggistiche dell'area veneta pone continuamente lo storico del paesaggio a tu per tu con opere e iniziative promosse da monaci.

Le fonti degli archivi monastici veneti sono dense e numerose: compravendite e affittanze, registri di contabilità e inventari di possedimenti, carte relative a controversie e migliaia di pergamene, rappresentazioni cartografiche e patti di bonifica, atti di investitura e registri di riscossioni. La memoria scritta dei monasteri offre

${ }^{2}$ Archivio di Stato di Padova, Corporazioni religiose soppresse, Monasteri del Territorio, S. Maria di Praglia, 165, c. 88v.

3 Queste opposte interpretazioni sono sostenute ad esempio da due grandi storici dell'economia rurale: Fumagalli (1994: 79-90) e Duby (1972: 108). Quanta «confusione» ingenerino nello storico le due diverse interpretazioni del ruolo dei monaci nel loro rapporto con il paesaggio è stato rilevato con molta franchezza da Bortolami (2001: 17). Si tenga presente, entro i confini del dibattito focalizzato sul "mito" dei "cistercensi dissodatori", anche Fossier (1983: 54-73). 
dunque un punto di osservazione privilegiato per seguire gli interventi di trasformazione del territorio portati avanti dal monachesimo ${ }^{4}$.

Muovendosi sulla scorta delle carte monastiche, alcuni luoghi del Veneto d'oggi sembrano riassumere profondità, riconnettendosi ad assetti paesaggistici mantenutisi per secoli, pur nei progressivi aggiustamenti, e infine cancellati con sorprendente velocità dalla storia contemporanea.

$\mathrm{Su}$ questa ricca base documentaria, in conclusione, è possibile avviare un'indagine che miri a una ricostruzione del paesaggio storico veneto e delle sue dinamiche formative, con il fine di agevolare, in un'ideale sovrapposizione tra passato e presente, una più compiuta e solida decifrazione dello stato odierno del Veneto, area regionale, come detto, dal punto di vista urbanistico e territoriale fittamente "sovrascritta" o, se preferiamo, "martoriata".

Prima di addentrarsi nelle porzioni del paesaggio veneto costruito dai monaci, occorre perimetrare il campo d'indagine. Prenderemo in esame gli ordini monastici testimoniati in area veneta il cui riferimento esplicito siano l'antica regola di san Benedetto o le regole da essa direttamente derivate: benedettini, cistercensi, camaldolesi e olivetani ${ }^{5}$.

Le sedi di questi ordini monastici censibili in Veneto dalle origini alle soppressioni napoleoniche risultano essere non meno di 260, all'incirca una ogni $80 \mathrm{kmq}$. Esse sono distribuite omogeneamente in tutto il Veneto, con l'eccezione dell'area montana del Bellunese. L'elenco comprende grandi abbazie e minuscoli priorati, cenobi con una storia millenaria e altri rimasti in vita per pochi decenni, monasteri dotati di una sterminata documentazione e altri di cui è appena nota l'esistenza.

Studiare in maniera approfondita i documenti di ognuno di questi monasteri lungo un millennio di storia non è possibile. Abbiamo dunque scelto come case studies una trentina di cenobi, in modo tale da coprire le diverse aree geograficoecologiche del Veneto ${ }^{6}$, e ristretto l'arco cronologico al periodo compreso tra il X e il XV secolo, ovvero all'epoca in cui l'azione di costruzione paesaggistica svolta

${ }^{4}$ Il panorama delle fonti archivistiche pertinenti ai monasteri veneti è letteralmente sconfinato. Inutile, nello spazio di una nota, avviare un censimento anche sommario dei fondi indagati e dei relativi luoghi di conservazione.

${ }^{5} \mathrm{Su}$ questi ordini, che ebbero in comune il riferimento alla regola benedettina ma che si differenziarono per genesi e talune interpretazioni della regola medesima, cfr. Rapetti (2013).

${ }^{6}$ Questo l'elenco dei monasteri selezionati: SS. Nazario e Celso (Verona), S. Giorgio in Braida, S. Zeno (Verona), S. Maria in Organo (Verona), S. Colombano (Bardolino), SS. Ilario e Benedetto (Dogaletto di Mira), S. Giorgio Maggiore (Venezia), S. Zaccaria (Venezia), S. Gregorio (Venezia), S. Giorgio di Fossone (Cavanella d'Adige), S. Michele Arcangelo Trinità di Brondolo (Sottomarina), SS. Felice e Fortunato (Vicenza), S. Vito (Vicenza), S. Croce di Campese (Bassano), S. Pietro (Villanova di San Bonifacio), S. Maria della Vangadizza (Badia Polesine), S. Pietro in Maone (Crespino), S. Maria (Mogliano Veneto), S. Eustachio (Nervesa), S. Maria Maggiore (Treviso), S. Maria (Follina), S. Bona (Vidor), S. Eufemia di Villanova (Villa del Conte), S. Daniele in Monte (Abano Terme), S. Stefano di Carrara (Due Carrare), S. Maria di Praglia (Teolo), S. Giovanni Battista del Venda (Vo'), S. Giustina (Padova). 
dai monaci fu più intensa e disegnò assetti del territorio destinati a perdurare nei secoli successivi ${ }^{7}$.

Le ricerche effettuate nei fondi archivistici dei monasteri veneti pongono di fronte centinaia di casi singoli, relativi a piccole e grandi operazioni di pianificazione e costruzione del paesaggio. La varietà documentaria, che restituisce uno dopo l'altro vividi scorci del paesaggio storico veneto, indurrebbe certo a intrattenersi fra queste carte con un gusto per certi versi "turistico", lasciandosi condurre da un luogo all'altro, dalle pianure alle lagune, dalle colline alle paludi, dai boschi ai prati foraggeri. Cercando meglio dentro questa stessa varietà, tuttavia, si possono ricavare i caratteri fondamentali dell'azione di costruzione e di modellamento del paesaggio perseguita in area veneta da abbazie e monasteri benedettini.

Monasteri e abbazie, al di là della dimensione religioso-spirituale, erano enti economici fondati sulla proprietà terriera. A seguito di processi di formazione patrimoniale molto articolati, essi giunsero a possedere superfici fondiarie estesissi$\mathrm{me} . \mathrm{Fu}$ in primo luogo per questa ragione che lo sforzo di pianificazione territoriale dei centri monastici non riguardò minuscole isole territoriali ma ebbe modo di distendersi su enormi superfici di suolo, segnando a fondo larghe porzioni del paesaggio veneto nel suo complesso.

La portata dell'azione monastica nelle campagne, perciò, si intuisce innanzitutto prendendo in esame le estensioni patrimoniali di alcuni cenobi. L'abbazia di Praglia, nel 1477, possedeva 887 ettari di terreni. Gli ettari di terre posseduti da S. Maria di Follina nel 1400 risultano essere 1287; erano invece 924, all'incirca nello stesso periodo, quelli della non lontana abbazia di S. Bona. S. Giustina di Padova, nel 1431, annoverava infine un patrimonio fondiario quantificabile addirittura in 1365 ettari $^{8}$. Si consideri, a titolo di paragone, come quella che è definita la «più vasta area industriale senza soluzione di continuità del Nordest», ossia l'area industriale di Padova facente capo al consorzio ZIP, si estenda oggi per 1050 ettari ${ }^{9}$ : all'incirca, dunque, quanto il patrimonio fondiario di una sola grande abbazia veneta del tardomedioevo.

Poiché l'agricoltura era il fondamento dell'economia di monasteri e abbazie, le iniziative monastiche nei confronti del territorio modellarono innanzitutto il paesaggio agrario. Uno sguardo di sintesi alla distribuzione delle proprietà dei monasteri veneti rivela l'esistenza di aree di radicamento fondiario privilegiato, nelle quali le proprietà si raccoglievano in grandi concentrazioni, e aree in cui le proprietà erano frammentate $\mathrm{e}$ isolate una dall'altra. Rispetto a quest'ultime, i maggiori cenobi si mossero secondo un fondamentale indirizzo: il compattamento fondiario. Nella documentazione consultata, infatti, si rileva la tendenza da parte dei monasteri ad accorpare particelle slegate e lontane tra loro, ora acquisendo i terreni intercalari fra una e l'altra mediante compravendite mirate, nel quadro di vere e pro-

${ }^{7}$ Si vedano a tal proposito almeno Rapetti (2012) e Rao (2015).

${ }^{8}$ Ricavo i dati, rispettivamente, da Gottardi (1989: 289); Archivio di Stato di Padova, Corporazioni religiose soppresse, Monasteri del territorio, S. Maria di Praglia, 52; Biblioteca Comunale di Treviso, ms. 110, De bonis monasterii Sanae Vallis; Archivio di Stato di Padova, Corporazioni religiose soppresse, Monasteri di Padova, S. Giustina, 17, cc. 97r-108v.

${ }^{9}$ Così si legge nel sito del Consorzio Zona Industriale e Porto Fluviale di Padova: http://www2.zip.padova.it/zip1.htm. 
prie campagne di acquisti, e ora, attraverso permute ben combinate, scambiando terreni isolati con terreni facilmente aggregabili a nuclei patrimoniali già localizzati in aree specifiche.

Questa politica di affermazione fondiaria fu di per sé una forma di costruzione del paesaggio; ma lo fu tanto più in quanto proprio in tali porzioni patrimoniali coese i monaci ebbero modo di concepire e di imprimere nel territorio una strutturale omogeneità paesaggistica ${ }^{10}$.

La compattezza raggiunta da molte proprietà monastiche non implicò tuttavia il configurarsi di un paesaggio "monocromo". Perseguendo una strategia agroeconomica che mirava a ottenere dalle proprietà una gamma il più varia possibile di prodotti, sia per l'uso interno dei cenobi che per la commercializzazione, i monasteri assecondarono le vocazioni microclimatiche e pedologiche delle singole proprietà e plasmarono un paesaggio agrario molto eterogeneo sul piano colturale e, per così dire, dell'impatto visivo.

Il differenziato paesaggio monastico, pertanto, fu in primo luogo un paesaggio i cui assetti furono segnati da scelte colturali. Non si dovrà pensare a estese monocolture, ma a zone in cui le varie opzioni agricole si presentavano intrecciate una all'altra, giustapposte $o$, al più, concentrate in aree definite a seconda dei potenziali rendimenti agricoli. Alla luce dei documenti, le colture portanti e sostenute dai monasteri erano la cerealicoltura (frumento, sorgo, orzo, miglio, segale e altri cereali minori), la viticoltura (oggetto di una costante implementazione, con precise strategie di estensione e di impianto di vitigni) e il prato da sfalcio (la cui incidenza nelle terre possedute dagli enti monastici veneti era molto varia, oscillante tra il $5 \mathrm{e}$ il $50 \%$ a seconda delle aree e delle possessioni) $)^{11}$.

Gli arativi cerealicoli, i vigneti e i terreni prativi costituivano certo gli elementi più ricorrenti dell'eterogeneo paesaggio agrario costruito dal monachesimo benedettino in area veneta, ma ad essi, oltre ad altre coltivazioni quali legumi, lino, riso, si affiancò potentemente l'arboricoltura. Vanno rilevate, in particolare, la coltivazione del castagno nell'area collinare dell'alto Trevigiano e l'olivicoltura praticata in aree che avrebbero in tal senso maturato una secolare vocazione: sui Colli Euganei, nel Bassanese e soprattutto nella zona del Garda, rispetto alla quale conosciamo terre monastiche in cui l'olivicoltura copriva il $66,2 \%$ delle superfici agricole disponibili (Castagnetti, 1972).

L'insofferenza alla sintesi univoca delle carte d'archivio, insomma, è speculare alla natura del paesaggio agrario costruito dai monaci. Esso non aveva standard fissi, generalizzabili, ma assetti diversi da luogo a luogo e da epoca a epoca, modellati tanto sulla base di esigenze di economia agraria quanto in sintonia con i contesti ambientali.

Se il paesaggio monastico venne indubbiamente caratterizzandosi in termini colturali, l'attività agricola promossa dai monasteri ebbe ricadute anche sul piano

${ }^{10}$ Questo dato si ricava da svariate fonti d'archivio, che riportano interventi più organici da parte dei monasteri proprio laddove i possedimenti erano più compatti. Più precisi rimandi alle fonti in M. Melchiorre, Il monachesimo benedettino e la costruzione del paesaggio veneto $(X-X V$ secolo), di prossima pubblicazione.

${ }^{11}$ Anche questo quadro è desunto dalla combinazione di numerose fonti d'archivio, il riferimento dettagliato alle quali non è possibile in questa sede. 
degli interventi edilizio-insediativi i quali, a loro volta, si tradussero in veri e propri iconemi paesaggistici, riferimenti visivi forti che connotavano il territorio riassumendone i soggiacenti rapporti culturali, economici e sociali ${ }^{12}$.

I più forti iconemi impressi dal monachesimo nel paesaggio veneto furono ovviamente i monasteri stessi, che costruirono paesaggio con la loro stessa fisica presenza. In alcune rappresentazioni cartografiche del XV secolo, i monasteri risaltano sistematicamente quali punti fermi di una trama paesaggistica, principi di orientamento geografico ed elementi che marcano il territorio ${ }^{13}$. Proprio in ragione di questa presenza forte, i monasteri rurali furono nodi di organizzazione territoriale e poli di aggregazione per l'insediamento umano nelle aree ad essi limitrofe. Allo stesso modo, ed è quest'ultima una tematica che meriterebbe una monografica attenzione, i cenobi urbani funzionarono spesso da nuclei poleogenetici: mediante veri e propri piani di lottizzazione, essi favorirono, pilotarono e controllarono le spinte espansive di alcuni settori delle città.

Tra gli apporti offerti dal monachesimo al tessuto insediativo del paesaggio veneto vanno quindi richiamati gli edifici rustico-abitativi. I monasteri, infatti, contribuendo non poco a infittire il vasto reticolo di case colonili che costituì, come noto, una componente caratterizzante e di lunga durata del paesaggio veneto toutcourt, incentivarono l'edificazione nelle proprietà agrarie di nuovi sedimina rurali, comprendenti edifici di abitazione, stalle, fienili, pozzi, forni. Particolare stimolo, peraltro, fu dato dal monachesimo, dietro importanti investimenti di capitale, all'edificazione di mulini, che costellavano con evidente regolarità le proprietà monastiche.

L'organizzazione del lavoro agrario nelle terre benedettine introdusse comunque nel paesaggio una modalità insediativa capace di imprimere una solida strutturazione antropica al territorio e di coagulare intorno a sé superfici agrarie e popolazione rurale: la curtis. Strumenti di accentramento delle proprietà terriere e del lavoro agricolo, che nelle terre monastiche del Veneto compaiono a partire dal IX/X secolo, le corti benedettine raccoglievano case, contadini residenti e servitù, spazi per l'attività molitoria e artigianale, ambienti per immagazzinare prodotti agricoli. Ad esse facevano capo possedimenti molto estesi e compatti, suddivisi in unità di conduzione (mansi) gestite in maniera organica. Sul modello delle corti benedettine, dal tardo XII secolo, si innestarono in seguito le grange cistercensi, complessi microcosmi economici che si segnalavano per uno spiccato accentramento della proprietà e del lavoro agricolo (Bortolami, 2001; Passolunghi, 2000).

Nelle corti benedettine prima e nelle grange cistercensi poi, in breve, veniva aggregata la popolazione rurale, si immagazzinavano prodotti, si centralizzava il lavoro agricolo e si realizzavano omogenei interventi sul territorio. Le curtes monastiche, perciò, incisero il paesaggio veneto perlomeno sotto due profili. Da un

${ }^{12}$ Sulla definizione degli iconemi cfr. Turri (1998, p. 170).

${ }^{13}$ Segnalo perlomeno la mappa del Quartier del Piave databile al secolo XV che ritrae l'abbazia di S. Bona di Vidor come un fermissimo punto di riferimento per tutta l'area (Archivio di Stato di Treviso, Corporazioni religiose soppresse, S. Bona di Vidor, b. 3) e la veduta a volo d'uccello del Padovano, opera di Nicolò Dal Cortivo (1534), nella quale spiccano, nei Colli Euganei, l'abbazia di Praglia e il monastero di S. Giovanni Battista sul monte Venda (Archivio di Stato di Venezia, Savi ed Esecutori alle Acque, Diversi, dis. 2).

166 
lato esse furono iconemi, segnarono il paesaggio, cioè, con la loro presenza. Dall'altro lato, e forse soprattutto, agevolarono con le proprie strutture immobiliari, economiche e sociali una più organica e razionale pianificazione del territorio, agendo da strepitosi incubatori di paesaggio agrario.

I monasteri veneti non possedevano unicamente terre floride, sulle quali si praticava un'agricoltura ben pianificata, ma anche terreni sterili, poveri, scarsamente produttivi. Nei confronti di queste terre marginali, i monaci non solo usarono un'attenzione non inferiore a quella usata nei confronti del territorio già messo a coltura, ma vi investirono risorse e sforzi se possibile ancora più ingenti, spingendo a fondo l'intervento di costruzione del paesaggio.

Nel rapporto dei monasteri con le porzioni dei loro patrimoni fondiari che potremmo riassumere sotto la categoria dell'incolto possiamo riconoscere quantomeno due sfaccettature, l'una "spirituale" e l'altra "materiale". Gli iniziali slanci mistici di non pochi cenobi avevano portato a cogliere proprio nell'incolto il paesaggio ideale in cui coltivare istanze eremitiche; molti monasteri di area veneta, perciò, si insediarono significativamente in paesaggi non antropizzati e tutelarono con cura lo spazio naturale interposto tra il cenobio e gli insediamenti umani, valorizzandolo nella sua sfuggente ma essenziale funzione di "frontiera spirituale"14.

In una prospettiva invece materiale, i paesaggi incolti restavano capitali fondiari che i monaci potevano e sapevano far fruttare. In primo luogo l'incolto era sfruttato per ciò che esso, in quanto tale, poteva offrire. Se le terre paludose, ad esempio, erano impiegate come pascoli, luoghi per la pesca o per l'approvvigionamento di strame, i boschi, che costituivano una parte considerevole dei patrimoni monastici, furono la base di un'economia del legname cui i monasteri veneti non mancarono di riservare specifica cura.

L'iniziativa monastica nei confronti dei terreni incolti non si limitò tuttavia allo sfruttamento passivo delle risorse che i medesimi incolti potevano offrire. Anzi: la trasformazione in spazi "produttivi" delle aree incolte e la loro valorizzazione agricola fu indubbiamente l'aspetto più eclatante della costruzione monastica del paesaggio. Fu nel recupero dell'incolto, infatti, che i monasteri cancellarono e riscrissero gli assetti territoriali di molte aree del Veneto storico.

Le carte d'archivio, a questo riguardo, conservano ben più che flebili tracce del diffuso lavorio promosso dal monachesimo benedettino. Ricordano bonifiche di suoli paludosi, cogliendo tra l'altro un importante elemento paesaggistico nelle terre di bonifica, ovvero i salici, essenza arborea atta al prosciugamento dei suoli paludosi. Parlano di estesi disboscamenti. Delineano il miglioramento di suoli tramite drenaggi e concimazioni. Registrano l'assunzione di manodopera per scavare canali e interrare valli palustri. Menzionano incendi mirati di boscaglia e sterpaglie. Descrivono lo sradicamento di cespugli, con conseguenti arature ${ }^{15}$.

${ }^{14}$ Sulle radici anacoretiche del monachesimo occidentale e sul frequente ritorno della fascinazione eremitica di un monachesimo aspro e solitario nei secoli medievali, cfr. Rapetti (2013, pp. 21-28; 97-106; 109-117).

${ }^{15}$ A questo riguardo gli archivi sono davvero ricchissimi di notizie; le referenze archivistiche ai documenti trovati in Melchiorre, Monachesimo benedettino. Quale studio esemplare di un'iniziativa di dissodamento e bonifica di un possedimento monastico d'area veneta, il rimando non può che essere a Varanini (1980-1981, pp. 39-142). 
Nelle terre contese ai boschi, alle paludi e ai suoli sterili, le opere di dissodamento portate avanti dai monaci non si accompagnarono soltanto alla valorizzazione agricola, ma anche, e in forme ancora più spiccate di quanto avveniva nelle terre già coltivate, all'incentivazione dell'insediamento umano. Anche questo, va da sé, ebbe rilevanti implicazioni sugli assetti del paesaggio veneto, sia agrario sia insediativo.

In primo luogo, benché si tratti di un fenomeno per sua natura frammentario e dunque piuttosto difficile da cogliere nella documentazione, all'estensione delle terre coltivate a danno dell'incolto corrispose il diffondersi di un insediamento rurale a dispersione, caratterizzato cioè dall'impianto di edifici rustici in singoli appezzamenti in via di dissodamento: case coloniche, fienili, stalle, tezze ${ }^{16}$. Dai documenti, in secondo luogo, emergono iniziative di pianificazione insediativa più articolate, volte alla fondazione nelle aree dissodate o da dissodare non di singoli edifici, ma di veri e propri villaggi di "pionieri" (come nel caso di S. Benedetto delle Selve ricordato in apertura) che furono al tempo stesso stimolo ed esito del recupero degli incolti ${ }^{17}$.

I documenti d'archivio, in conclusione, non lasciano dubbi circa la pervasività con cui i monasteri veneti, nel corso dei secoli, aggredirono estensioni di terra incolta, si trattasse di piccoli appezzamenti o di grandi possessioni fondiarie, di boschi o di paludi, di terre magre o di aree ghiaiose, di versanti collinari o di aree dissestate dal punto di vista idrogeologico, di porzioni di pianura o di instabili superfici lagunari. Nella costruzione del territorio veneto, in altre parole, il monachesimo, pur accanto ad altre forze (i comuni cittadini o rurali e i grandi possessori laici prima, i grandi casati signorili della Terraferma e la Repubblica di Venezia poi), giocò un ruolo primario tanto nell'organizzazione e nella razionalizzazione del paesaggio agrario quanto nella trasformazione dei paesaggi incolti in paesaggi agrari e terre abitate.

Nella loro plurisecolare costruzione paesaggistica i monaci appaiono alla stregua di intelligenti registi, capaci non solo di concepire ex nihilo le iniziative, ma anche di recepire proposte provenienti dall'esterno, di assecondare i processi economico-sociali in atto nel campo dell'agricoltura e dell'insediamento, di coordinare, secondo disegni di vasta scala, gli sforzi quotidiani dell'eterogenea fascia di popolazione rurale che si trovava a condurre terre di proprietà monastica. Il precipuo quadro valoriale dell'operare monastico in un ambiente concepito come naturale, umano e divino al tempo stesso è cosa che non si discute; tuttavia, seguendo la

${ }^{16}$ Un esempio duecentesco particolarmente efficace, relativo a una iniziativa di questo tipo promossa dall'abbazia di Follina, in Archivio di Stato di Venezia, San Michele in Isola, Pergamene, 18, perg. 09.09.1290.

${ }^{17}$ Gli esempi citabili sono piuttosto numerosi. Limitiamoci a menzionare le colonizzazione insediative avviate in Asiago dal monastero vicentino dei SS. Felice e Fortunato nel XII secolo (Bortolami, 1994); l'insediamento di Franchavilla merchatorum, poco a valle di Badia Polesine, fondata con il sostegno del comune di Verona ma con il diretto coinvolgimento dell'abbazia della Vangadizza (Rigon, 1985); il paese di bonifica di Villa del Bosco, nel Padovano, la cui nascita si deve all'intraprendenza dell'abbazia di S. Giustina (De Sandre Gasparini, 1979) e Villanova Istrana, nata sullo scorcio del secolo XII per iniziativa delle monache di S. Maria di Mogliano (Bortolami, 1999, p. 130). 
traccia della documentazione d'archivio, è innegabile che i monaci furono costruttori vincenti di paesaggio in primo luogo in quanto "imprenditori" agrari, interessati a ottimizzare e a incrementare i rendimenti dei propri capitali fondiari.

Nella direzione dei processi che plasmarono vaste porzioni del paesaggio storico del Veneto, l'impatto concreto dell'attività dei monaci variò a seconda di diversi fattori e innanzitutto delle modalità usate nella gestione delle proprietà fondiarie. Quali che fossero le strategie gestionali, tuttavia, la costruzione del paesaggio favorita dai monaci era di fatto condivisa con i "contadini”, a tutti gli effetti coprotagonisti nella pianificazione e nel modellamento del territorio.

I monaci furono dunque registi e i contadini esecutori; senza gli uni o senza gli altri la costruzione di paesaggio nelle terre dei monasteri non avrebbe raggiunto le proporzioni e l'efficienza che le fonti storiche permettono di intuire. E benché gli archivi monastici, per loro stessa natura, disegnino una prospettiva che fa più luce sui registi che sugli esecutori, taluni documenti hanno la forza di riportare in primo piano anche il mondo rurale, una folla sterminata di contadini, braccianti, coloni, fittavoli. L'incisiva e raffinata costruzione del paesaggio portata avanti dai monasteri veneti nel corso dei secoli, insomma, fu debitrice alle fatiche e al lavoro di migliaia di anonimi contadini non meno che alla razionalità organizzativa che fu propria degli enti monastici.

Resta da chiedersi, in conclusione, se il monachesimo benedettino avesse non tanto una progettualità nella gestione del territorio, il che è evidente, ma se avesse, in questo, una sensibilità sua propria. Le carte d'archivio testimoniano che era certo in gioco una sensibilità specifica, nutrita degli ideali mistico-spirituali propri del monachesimo; ma quest'ultima pur ravvisabile attitudine, quanto a concreto modellamento del territorio, ebbe un peso inferiore rispetto alla marcata sensibilità agronomico-economica dei monaci.

Quest'ultimi, infatti, sotto la spinta di contingenze specifiche e mettendo a frutto un'effettiva conoscenza ambientale, agirono nella prassi senza mai perdere di vista i fini materiali. La costruzione del paesaggio da parte dei monaci, insomma, al di là dei facili slogan, più che una vocazione a una pianificazione territoriale "illuminata", fu una risposta efficace, concreta e ben organizzata, in dialogo accorto e competente con l'ambiente, a esigenze di ottimizzazione patrimoniale: migliorare e incrementare le rendite dei campi, convertire l'incolto improduttivo in coltivo redditizio, limitare i danni degli agenti naturali, trovare soluzioni agricole capaci di far aumentare gli introiti, effettuare investimenti di capitale in grado di garantire precisi ritorni finanziari.

I documenti ci mettono di fronte, in altre parole, a principi aziendali più che a principi di pianificazione astratta, o, se proprio vogliamo, a una pianificazione territoriale che nei suoi risvolti pratici non fu che l'effetto derivato di una programmazione di natura economica. Ne risultarono fisionomie, dinamiche e concezioni dello sviluppo del territorio ben precise. Con gli orientamenti che in epoche sensibilmente più recenti hanno dato forma al paesaggio veneto contemporaneo esse condividono certo il principio di fondo: al di là dei criteri estetici, l'ambiente va pensato e costruito in base alle esigenze socio-economiche di chi lo controlla e/o lo possiede.

Profondamente diversi, però, furono i termini con cui i monaci tradussero in pratica questo principio di fondo. Il tratto saliente della costruzione monastica del 
paesaggio veneto, infatti, è l'assenza di uno standard generalizzato e imposto sistematicamente in ogni luogo. La logica di sviluppo territoriale seguita dagli enti monastici, che al caso seppe essere "violenta" e impattante, era sì quella di rendere produttivo e redditizio l'ambiente naturale, ma dialogando con esso, integrando la progettualità nei contesti specifici, secondo un reciproco (e proficuo) condizionamento.

Le tracce di questa calibratissima logica e dell'incessante sforzo di costruzione territoriale sostenuto dai monaci si possono leggere ancora oggi, sepolte e assediate dalla "città diffusa", negli interstizi del Veneto contemporaneo. E comunque un paesaggio pressoché invisibile, i cui frammenti sopravvivono appena, galleggianti in un tessuto urbanistico caotico $o$, al più, in un paesaggio agrario urbanizzato. Attraverso la memoria scritta, tuttavia, il paesaggio invisibile modellato dai monaci, e dai contadini di quest'ultimi, può essere ben più che intravisto e, perché no, avere ancora qualcosa da insegnare.

\section{Riferimenti bibliografici}

Zanzotto A. (1999). Le poesie e prose scelte (a cura di Dal Bianco S. e Villalta G.M.). Milano: Mondadori.

Zanzotto A. (2009). Conglomerati, Milano: Mondadori.

Zanzotto A. (2013)., Luoghi e paesaggi (a cura di M. Giancotti). Milano: Bompiani.

Trevisan V. (2002). I quindicimila passi, Torino: Einaudi.

Trevisan V. (2016), Works, Torino: Einaudi.

Vallerani F. e Varotto M. (2005) (a cura di). Il grigio oltre le siepi. Geografie smarrite e racconti del disagio in Veneto, a cura di Portogruaro (VE) 2005;

Vallerani F. (2013). Italia desnuda. Percorsi di resistenza nel Paese del cemento. Milano 2013.

Indovina F., Matassoni F., Savino M., Sernini M., Torres M. e Vettoretto L., La città diffusa. Venezia: Daest-IUAV

Savino M. (1999). Città diffusa; reti; ambienti insediativi: la ricerca di una verosimile definizione dei processi di trasformazione del territorio. In: Indovina F., a cura di, Territorio. Innovazione. Economia. Pianificazione. Politiche. Vent'anni di ricerca del Daest. Venezia: Daest-IUAV, 61-81.

Munarin S. e Tosi M.C. (2001). Tracce di città. Esplorazioni di un territorio abitato: l'area veneta. Milano: FrancoAngeli.

Indovina F. (2009) (a cura di). Dalla città diffusa all'arcipelago metropolitano. Milano: FrancoAngeli.

Taine H.A. (2003). Viaggio in Italia, Torino: Aragno (ed. orig. 1817).

Goethe J.W. (1983). Viaggio in Italia. Milano: Mondadori (ed. orig. 1866).

Fumagalli V. (1994). Monaci contadini, in Idem, Paesaggi della paura. Vita e natura nel Medioevo.Bologna, 1994.

Duby G. (1972), L'economia rurale nell'Europa medievale. Francia, Inghilterra, Impero (secoli $I X-X V)$. Bari: Laterza.

Bortolami S. (2001). "Corti" e "granze" benedettine nel medioevo: alle origini di una storia di lunga durata. In: Vita M. e Trolese F.G.B., a cura di, La corte be- 
nedettina di Legnaro. Vicende, strutture, restauri. Venezia-Legnaro: Veneto Agricoltura.

Fossier R. (1983). L'économie cistercienne dans les plains du Nord-Ouest de l'Europe. In: L'économie cistercienne. Géographie. Mutations du Moyen Âge aux Temps modernes, Auch: Comité départemental du tourisme du Gers.

Rapetti A. (2012). La terra degli uomini. Campagne dell'Italia medievale. Roma: Carocci Editore.

Rapetti A. (2013). Storia del monachesimo medievale. Bologna: il Mulino.

Rao R. (2015). I paesaggi dell'Italia medievale, Roma: Carocci Editore.

Gottardi M. (1989). L'incontro tra un'abbazia in declino e una famiglia in ascesa. Gli Erizzo a S. Bona. In: Gasparini D., a cura di, Due villaggi della collina trevigiana. Vidor e Colbertaldo, III: L'età moderna (secoli XV-XVIII). Vidor: Comune di Vidor.

Castagnetti A. (1972). I possessi del monastero di San di Verona a Bardolino. Studi medievali, 13: 97-159.

Turri E. (1998)., Il paesaggio come teatro. Dal territorio vissuto al territorio rappresentato. Venezia: Marsilio.

Passolunghi P.A. (2000). Esperienze agrarie in ambito monastico lungo il Piave: la granza di Sottoselva. In: Trolese F.G.B., a cura di, Mogliano e il suo monastero. Mille anni di Storia. Atti del Convegno di Studi, Mogliano Veneto, 6-7 giugno 1997. Cesena: 152-153.

Varanini G.M. ((1980-1981). Un esempio di ristrutturazione agraria quattrocentesca nella "Bassa" Veronese: il monastero di S. Maria in Organo e le terre di Roncanova. Studi storici veronesi Luigi Simeoni, 33-31: pp. 39-142.

Bortolami S. (1994). L'altipiano nei secoli XI-XIII. Ambiente, popolamento, poteri, in Storia dell'Altipiano di Asiago In: Stella A., a cura di, Territorio e istituzioni. Vicenza: Neri Pozza e Accademia Olimpica, 270-279);

Rigon A. (1985). «Franchavilla mercatorum». Mercanti veronesi, abbazia della Vangadizza e un'impresa di bonifica nel primo Duecento. Archivio Veneto, 124: 5-37.

De Sandre Gasparini G. (1979). Contadini, chiesa, confraternita in un paese veneto di bonifica. Villa del Bosco nel Quattrocento, Padova: Istituto per la storia ecclesiastica padovana.

Bortolami S. (1999). Il monastero di S. Maria di Mogliano e le comunità rurali del Trevigiano nel Medioevo. In: Bortolami S., Chiese, spazi, società nelle Venezie medioevali, Roma 1999, p. 130). 